\title{
Encouraging Brain Injury Rehabilitation through Ludic Engagement
}

\author{
Rachel McCrindle ${ }^{1}$, Stephen Simmons ${ }^{1}$, Richard Case ${ }^{1}$, Malcolm Sperrin ${ }^{2}$, \\ Andy $\mathrm{Smith}^{3}$, and Carol Lock ${ }^{4}$ \\ ${ }^{1}$ School of Systems Engineering, University of Reading, Reading, UK \\ ${ }^{2}$ Department of Medical Physics, Royal Berkshire Hospital, Berkshire, UK \\ ${ }^{3}$ Department of Clinical Engineering, Royal Berkshire Hospital, Berkshire, UK \\ ${ }^{4}$ Headway Brain Injury Association, Thames Valley, UK \\ r.j.mccrindle@reading.ac.uk
}

\begin{abstract}
Whilst in hospital immediately following a stroke or other acquired brain injury, patients receive, and engage in, a structured, concentrated and supervised programme of rehabilitation. However, once they leave hospital patients frequently fail to engage in the rehabilitation exercises provided for them. This paper describes how the Microsoft Kinect sensor has been used with computer games to engage patients with their rehabilitation following stroke and other brain trauma injuries. Initially off-the-shelf games were used, the ludic nature of the games, masking the treatment element of the exercises. However, whilst this approach was a great success in terms of patient engagement it was found that off-the-shelf games were frequently too fast or too complex for some patients to play and set-up due to the extent of their brain traumas. To address these issues, a system, PURR (Prescription Software for Use in Recovery and Rehabilitation), has been developed that uses the same ludic principles to enagage patients whilst allowing games to be tailored to a patients condition, requirements and interests.
\end{abstract}

Keywords: Ludic engagement, Kinect, brain trauma, stroke, recovery and rehabilitation, case study, personalization, usability.

\section{Introduction}

Acquired brain injury refers to all circumstances in which brain injury has occurred since birth, and includes traumatic brain injuries caused by incidents such as road traffic accidents, assaults and falls, as well as injuries acquired through conditions such as tumor, stroke, brain hemorrhage and encephalitis. The effects of a brain injury can be wide ranging, and depend on a number of factors such as the type, location and severity of injury. Every person's injury is unique, so they will experience any number of symptoms, which can range from mild to severe [1]. The resulting damage caused by a stroke or other types of acquired brain injury can be very widespread and longlasting often requiring rehabilitation both in the hospital and after discharge. Statistics indicate that there are in excess of 1 million people in the UK and 5.3 million people 
in the US living with long-term effects of brain injury [2, 3]. Putting these figures further into perspective 558 per 100,000 UK residents will sustain a brain injury, with someone in the UK being admitted to hospital with an acquired brain injury every 90 seconds. Nor is brain injury restricted to older adults. Whilst over 80 year olds are a major risk group, anyone can acquire a brain injury, with for example, one of the groups most at risk groups being 15-24 year old males [2]. The need to find ways of cost effectively and engagingly providing routes to recovery and rehabilitation that can be continued after discharge from hospital is a pressing one. The work described in this paper is collaboration between the University of Reading, the Royal Berkshire Hospital and Headway Brain Injury Association. It has focused on the use of the Kinect as a supplementary treatment to traditional rehabilitation and continued assessment of patients following a stroke or other serious brain injury, initially using off-the-shelf games and subsequently a system that can deliver bespoke rehabilitation programmes tailored to a patient's interests, condition and abilities, both in the hospital and after patients are released.

\section{Games for Therapy}

The use of games and/or gamification for therapy and rehabilitation has been gaining momentum over the past decade. Not only has there been a realization of the power of games to engage and motivate players, but the advent of social networking and causal gaming has broadened the market for and acceptance of gaming in general [4]. The plethora of gaming devices now available and the play anytime, play anywhere, nature of many games has made gaming a ubiquitous pass-time. More natural and accessible ways of interacting with games coupled with more affordable pricing structures has led to a mass uptake of gaming technology by the general population. Games are no longer just for children or young adults they can be played and enjoyed by all age groups [4].

Games and their ludic elements have been used in many health-related practices including pain distraction, cyberpsychology, disease management, health education and rehabilitation [5, 6]. Ludic engagement may be considered as a way of blurring the distinctions between 'work' and play [7] such that participants are motivated by curiosity, exploration, and reflection rather than the externally-defined tasks [8]. In a health context this is seen as patients interacting with and becoming so engrossed in the gameplay that they perceive it to be a fun and inclusive experience, rather than a set of repetitive exercises. This encourages them to participate more regularly and to engage for longer periods of time with their therapy especially when they leave the hospital environment and continue therapy at home.

The application of entertainment technology to stroke and brain injury rehabilitation has been boosted by the invention of home based gaming technologies such as the Nintendo Wii [9], PlayStation Move [10] and Microsoft's Kinect [11] which, with their natural user interfaces (NUIs), have made it easier for people of all ages and with varying health conditions, disabilities and technical know-how to interact with computer games. The Kinect, a motion sensing input device capable of tracking the 
whole human body without the need for the user to hold any physical device, together with its ability to collect a vast amount of data about skeletal positioning, motor control and game play interaction has become of particular interest for use in brain injury rehabilitation and recovery $[12,13,14,15]$.

In this paper we report on a study into how concepts of gamification and ludic engagement [8] have been combined with more traditional physio/occupational therapy exercises in order to engage patients in brain injury recovery and rehabilitation at the Royal Berkshire Hospital and Headway Brain Injury Association. How this approach has aided one patient is described in detail in the paper. Analysis of their experience together with feedback from other patients and physio/occupational therapy staff regarding the use of off-the-shelf games for rehabilitation purposes has led to the development of a system, PURR (Prescription Software for Use in Recovery and Rehabilitation), which can deliver treatment programmes tailored to an individual patient's condition, requirements and interests.

\section{Study to Assess Engagement of Brain Injury Patients with the Kinect}

In the summer of 2012, the Royal Berkshire Hospital (RBH) initiated a study which used 'off-the-shelf' Xbox games as a supplementary treatment to traditional rehabilitation for stroke patients, both in the hospital and after patients were released (Fig.1).
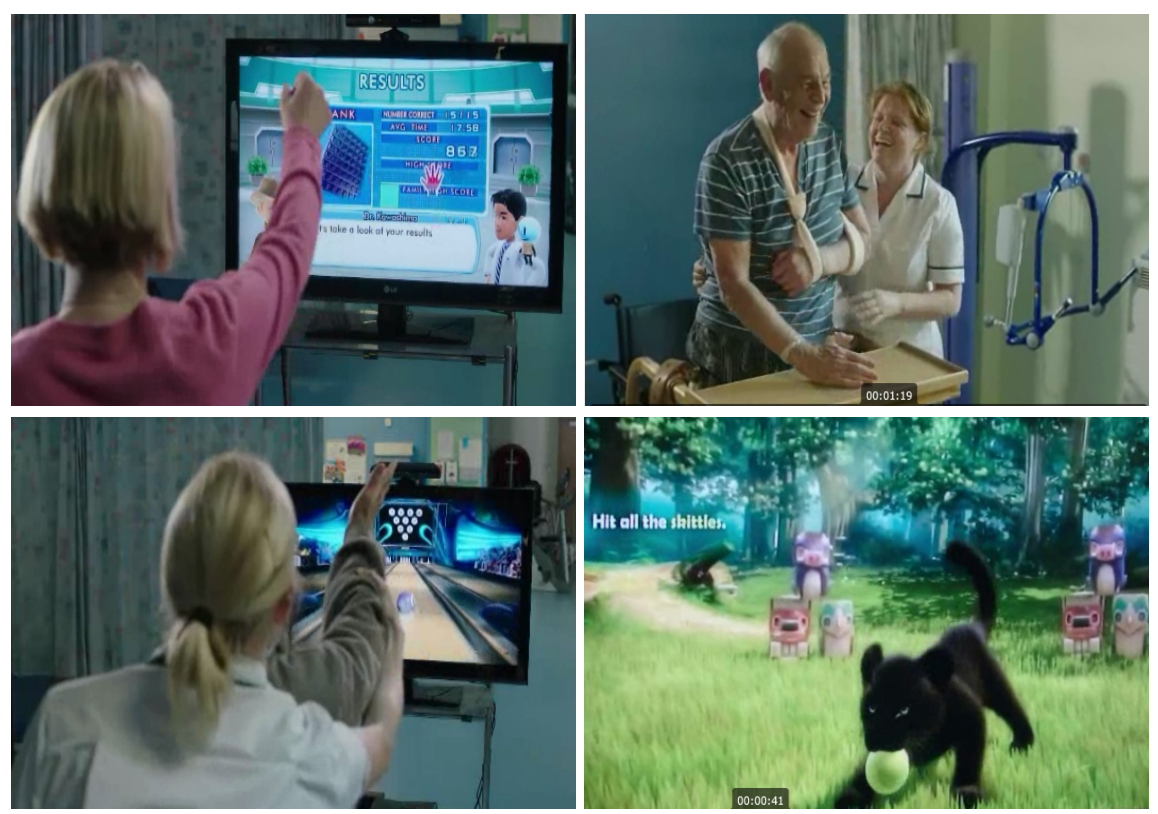

Fig. 1. Patients engaging with off-the-shelf Kinect games during study [17] 
This approach to rehabilitation proved to be highly effective with regards to patients' engagement in and enjoyment of therapy [17]:

“... a very big part of rehabilitation is trying to enjoy what you do and a sense of achievement gives you that sensation ..." Vipal Thaker, Patient Royal Berkshire Hospital

It was also seen to have the effect of making therapy more inclusive by involving other members of the family in the same activities:

“... at home it can be a good family gathering and at the same time helping you regain your mobility and balance ..." Vipal Thaker, Patient Royal Berkshire Hospital.

Therapists also saw the value of including Kinect-based games within their therapy sessions:

“... can note the improvement in their score and how that translates into what they can do for themselves in terms of their standing balance and arm strength ... and how this translates into them becoming more independent ..." Vicky Morris, Occupational Therapist, Royal Berkshire Hospital.

As part of the same study the Kinect and Xbox 360 were also used extensively with the clients of Thames Valley Headway Brain Injury Association, who had varying symptoms resulting from acquired brain injuries. As indicated, the nature of brain injuries and their impact is different for every person, however the case study reported in Section 4 is typical of patient interaction with, and responses, to the introduction of off-the-shelf Kinect games as part of their treatment.

\section{Case Study - Therapy Utilising the Kinect}

In order to determine if the ludic nature of the Xbox and Kinect sensor can engage a patient and encourage them to follow a post-hospital rehabilitation programme, a number of follow up interviews were undertaken with patients who had been given a Kinect/Xbox to use in their own homes. One such case is described below.

\subsection{Description/Pathology}

The patient is a gentleman in his mid thirties, married with two young children. For the purpose of this paper he will be referred to as Mr P. Due to this patient's communication difficulties, all the following information has been gathered from his spouse.

Mr P. sustained a traumatic head injury some eight years ago which resulted in a lengthy hospital stay followed by nine months as an inpatient on the Putney Rehabilitation Unit. Previous to his injury, Mr P. was in full time employment and his leisure activities included walking, going to the gym and playing computer games.

As a direct consequence of his head injury $\mathrm{Mr} \mathrm{P}$ was left with the following residual deficits: 
- Dense right sided hemiplegia

- Neglect of right upper limb

- Flexed deformity to his right elbow

- Extensor pattern in right lower limb

- Poor gait pattern

- Reduced mid line awareness

- Receptive and expressive disphagia causing significantly problematic communication

- Impulsivity

- Lack of initiation

- Lack of motivation

- Reduced confidence

\subsection{Formal Rehabilitation}

Whilst on the brain injury rehabilitation unit in Putney, Mr P. received both occupational therapy and physiotherapy input. Occupational therapy was on a daily basis and physiotherapy two to three sessions per week.

Although he engaged well with occupational therapy (as it had immediate meaning to him in his activities of daily life) $\mathrm{Mr} \mathrm{P}$. was difficult to engage in the physiotherapy sessions due to his cognitive changes. He found it a challenge to understand their significance, as to him, the exercises were 'just exercise' and bore little relevance to everyday life. Post injury, he was unable to appreciate that the programmes were designed to physically enable him to achieve independence with his ordinary daily needs. Mr P. would only engage in physical activity when instructed to do so and had no carryover of information to enable him to undertake exercises independently. Lack of motivation would appear to be another of the significant issues in his reluctance to participate as the exercises he was required to practise were presented in a manner that was too abstract for him to appreciate.

\subsection{Post Structured Rehabilitation}

Although Mr. P. was discharged home with a physiotherapy programme to undertake with family members, engagement was difficult due to already mentioned factors. Despite much effort from the family, Mr P's wife believes that for him to engage in formal physiotherapy exercises they would need to be undertaken within a rehabilitation setting as this was where he would associate some relevance. As a direct consequence, Mr P. would not do his physiotherapy exercises within the home unprompted

\subsection{Introduction of the Xbox in the Home Environment}

Mr P. was introduced to the Xbox Kinect approximately 6 months ago, when, on a typical day would spend $99 \%$ of his waking day sat in a chair, unchallenged and unmotivated to partake in any physical activity. 
Initially he would begin a game but would fatigue and rest again after 5-10 minutes, as although enjoying the participation he was unused to physical exertion. His stamina has now greatly improved and nowadays he is able to play for up to 2 hours before tiring. Although, due to his cognitive and physical limitations he is unable to set up to play, he will ask for someone to set up for him, this is one of a very few things Mr P. will initiate.

In a direct comparison to his formal physiotherapy regime, Mr P never needs reminding to use the Xbox Kinect and his engagement has shown many positive benefits. He is now able to socialise. As Mr P. has very limited speech and has physical limitations, he was unable to engage in meaningful discussion with the family nor was he motivated to play with his children. Since the introduction of the Kinect system he has demonstrated vocal improvements as he's having to teach the children certain things about the games. It has given him a new role. Mr P. is now able to fully share an interest with other members of the family that doesn't make him feel 'different', as they all share the same experience with each other.

There have also been improvements made in his ability to sustain and switch his attention. Whilst playing, he is constantly having to maintain and adapt his focus to achieve better scores.

Confidence is another area of improvement. This has grown out of teaching his children how to play the games and them looking to him for advice and guidance.

Both sitting and standing balance ability has increased, Mr P now has improved mid line awareness and can self correct when prompted whereas, before the introduction of the Xbox Kinect he would always favour his unaffected left side. Mr P. now engages the use both of his upper limbs to play the games and his right lower limb has increased in strength, enough to rely upon it in dynamic standing.

The question of engagement with the Xbox Kinect within the hospital setting was put to the spouse of Mr. P. As he sustained his injury 8 years previously, the console was not available, however, she interestingly reported that, had Mr P. used the Xbox within the acute setting, he would have associated its use at home with his hospital stay. She felt that this would have had a negative bearing on Mr P.'s acceptance and use of it within the home.

\subsection{What Modifications Could Be Made to Make the X Box Kinect More User Friendly to the Study Group?}

This client, as others, has difficulty with the setting up of the console and individual games. He is unable to type in names as he can't maintain a steady upper limb and finds it too difficult to use his hand in the correct position to select options. Thus far, he only tends to play when his wife is present to set up a programme on his behalf, if this was made easier for him to achieve independently she is confident that he would learn how to do this for himself. Mrs P. also fed back that the whole set up process at present is too lengthy, by the time Mr P. had gone through all of the separate stages he would probably give up on playing the game itself.

At present, there are only a limited number of games that Mr P. can participate in fully, his main difficulty with the more 'active' games such as Rapids is that he is 
unable to jump or move quickly enough to gain scores. It was suggested that perhaps the speed of the games could be adjustable to suit varying physical abilities, increasing as the player was able.

Although Mr P. is able to stand, it was also suggested that there could be games designed around wheelchair users who are only able to use their upper bodies.

\subsection{Discussion}

$\mathrm{Mr} \mathrm{P}$. is unable to fully appreciate the physical and cognitive improvements he has made in this relatively short time since his introduction to the Xbox Kinect, therefore, as before, we have gathered the summary of information from his wife.

Mrs P. reports that her husband has become better able to communicate and connect with his children and members of his extended family since being able to use the console. $\mathrm{He}$ is re engaging in aspects of family life and has a new, positive role to play as a father. In her own words: 'It is a joy to see'.

His ability to concentrate on tasks has improved and he is making efforts to verbally communicate. His physical improvements are various and positive. He is able to use his upper limbs bilaterally when playing the games, he doesn't need to think about trying to do this, he just does it. Awareness of his mid line is more automatic, the strength in both upper and lower limbs has increased and both his sitting and standing balance has improved.

Mr P. regards the Xbox Kinect as a recreational pursuit and does not perceive the exercise he is undertaking to be an alien concept as he does the formal physiotherapy programmes. He is having fun, improving his ability to concentrate, becoming fitter and is motivated to engage in something that involves a physical challenge.

\section{PURR System}

Despite receiving a positive response from many patients especially with regards to making their treatment more fun and engaging, feedback, like that from Mr P., also showed that off-the-shelf games were frequently too complex and 'fast' to play for many patients, and were difficult to set up.

Additionally, whilst the scoring system of an off-the-shelf game provides a goal for patients to exceed, from a clinical point of view off-the-shelf games can only provide subjective results, as scores are not truly representative of improvement and therefore have limited medical benefit for healthcare professionals in assessment of patient progress.

In order to address these issues, the PURR (Prescription Software for Use in Recovery and Rehabilitation) system has been developed in consultation with occupational therapists and physiotherapists who defined the metrics they need collected from the system in order to assess patient progress, and with potential users of the system, who had suffered different types and severity of brain injuries, with regards to developing games tailored to their abilities, interests and rates of recovery. PURR can be used with patients at all stages of their neurological injury rehabilitation, in hospital and at home. It comprises two key components [18]: 
1. A games development platform utilizing the Microsoft Kinect that can be used to create engaging and clinically relevant Patient Rehabilitation Experiences (PREs) tailored to a patients abilities and improvement of their clinical symptoms, as well as to their personal interests (Fig 2 left).

2. A metrics and monitoring engine for collecting data that can be used to track and assess the progress a patient is making as a result of their rehabilitation programme, and for adjusting in real-time game play to a level that suits their capabilities (Fig 2 right).
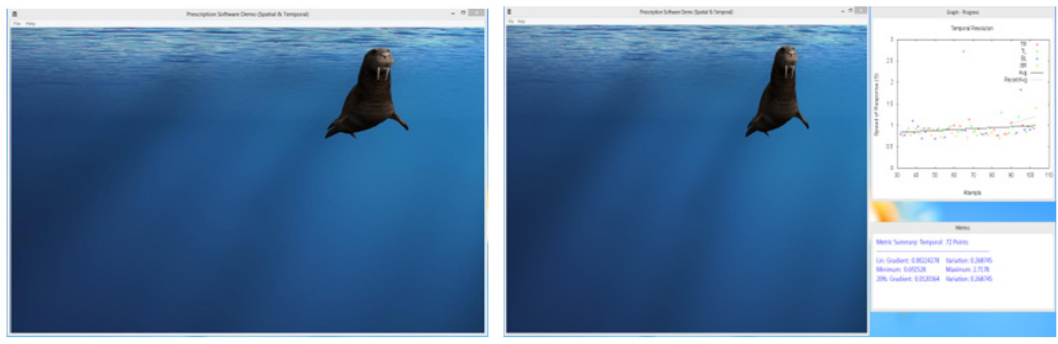

Fig. 2. Example of Patient's view (left) and Therapist's view (right) of screens
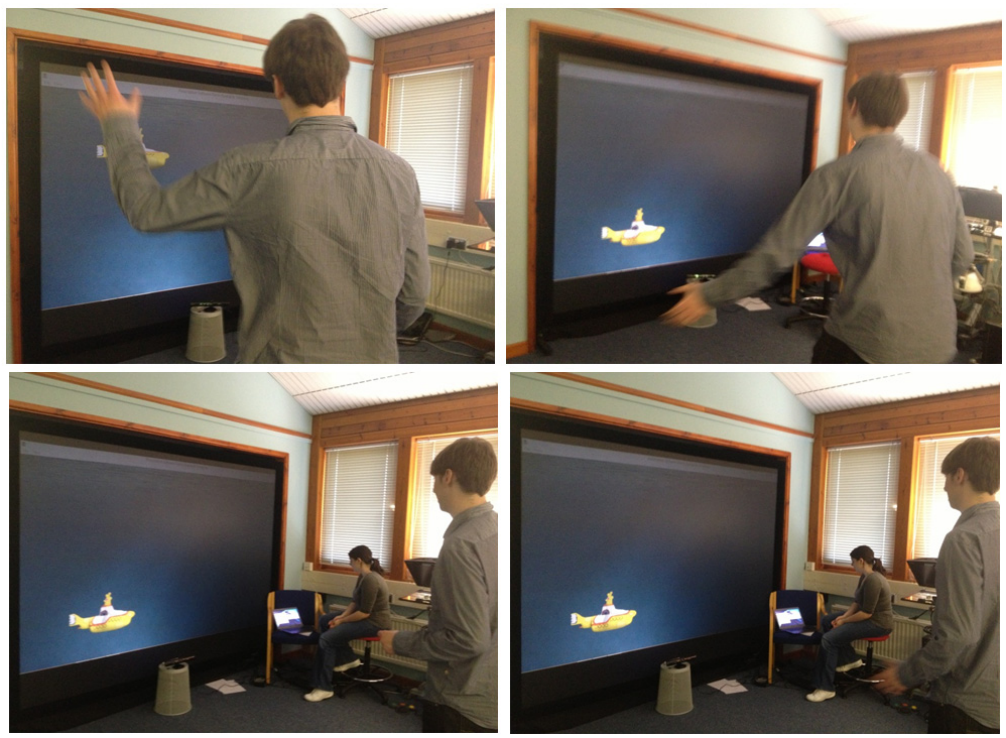

Fig. 3. Interacting with the game

The PREs operate as mini-games, challenging the patient to perform activities normally limited by their injuries encouraging them to perform better than they had previously thought possible and facilitating recovery (Fig 3). Feedback is given instantly for exceeding goals. Each experience dynamically alters to the abilities of a patient 
such that if a patient struggles to complete an action in a timely manner the next similar iteration will be simplified, thereby providing challenges that scale with ability and progress. Additionally, as the demographic of patients with brain injuries is wide, ranging across ages, backgrounds and interests, the PREs can be configured to be visually appealing and engaging to a specific patient and can be played whether standing or sitting. The ability for the software to self-adjust gameplay parameters based on a person's abilities means that rehabilitation can become a family-centred activity, something that was seen to be very important by many patients including $\mathrm{Mr} \mathrm{P}$ (Fig 4).
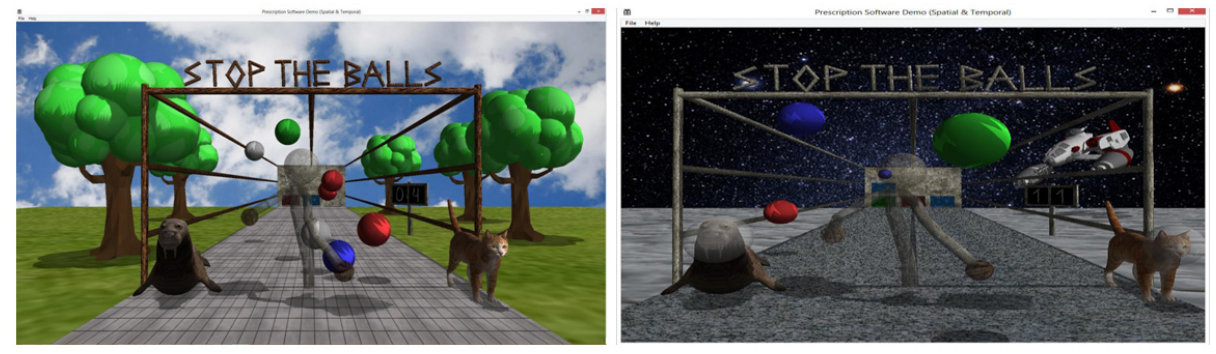

Fig. 4. Bowling game customized for field or space scenario

\section{Summary}

In trials utilizing off-the-shelf games and the personalised Patient Rehabilitation Experiences (PREs) we have consistently seen how the ludic nature of games can change patients' perceptions of the treatments they are receiving from being repetitive exercises to entertaining experiences. By making the recovery and rehabilitation process a fun, engaging, personalised and family-centric activity, in comparison to traditional rehabilitation exercises which are often repetitive and with no immediate feedback, we found that patients were more likely to not only engage in their treatment when a therapist was present, but also to initiate therapy sessions themselves when within their own homes and outside of therapist visits.

However, whilst off-the-shelf games were considered to be fun and engaging many patients were unable to make full use of the game features due to their complexity and speed of play, as well as the need for more tailored gameplay to suit their particular therapy needs. Additionally off-the-shelf games did not provide enough data to be able to clinically assess patients' progress. With these considerations in mind we developed the PURR ((Prescription Software for Use in Recovery and Rehabilitation) system.

Use of the PURR system to date has been well received by patients and health professions. The system is currently being enhanced and expanded to encompass further PREs, personalization, metrics collection and medical conditions. Larger scale trials are underway, as are trials with particular individuals over longer periods of their rehabilitation. The new Kinect 2 sensor is also being integrated into the PURR system 
in order to exploit its greater sensitivity and features with regards to providing more accurate tracking and a larger data set that can be analyzed to provide therapists with more precise assessments of patient progress.

Acknowledgements. We are very grateful for the contributions made and evaluations undertaken by the staff and patients of the RBH and Headway.

\section{References}

1. Headway, About Brain Injury (2014), https: / / www . headway . org.uk/About-Brain-Injury.aspx

2. Headway, Brain Injury Statistics (2014), https://www.headway.org.uk/key-facts-and-statistics.aspx

3. Brain Injury Association of America, Brain Injury Facts (2014), http: / / www. biausa.org/LiteratureRetrieve.aspx? ID =104992

4. McCallum, S.: Gamification and Serious Games for Personalilised Health. In: Blobel, B., et al. (eds.) pHealth 2012, pp. 85-95. IOS Press (2012)

5. Sawyer, B.: From Cells to Cell Processors: The Integration of Health and Video Games. IEEE Computer Graphics and Applications 28(6), 83-85 (2008)

6. Peterson Brooks, E.: Non-formal Learning through Ludic Engagement within Interactive Environments. Doctoral Thesis, Malmo University Electronic Publishing (2006), http: //dspace.mah. se/handle/2043/7970

7. Lindley, C.A.: Ludic Engagement and Immersion as a Generic Paradigm for HumanComputer Interaction Design. In: Rauterberg, M. (ed.) ICEC 2004. LNCS, vol. 3166, pp. 3-13. Springer, Heidelberg (2004)

8. Gaver, W.W., Boucher, A., Bowers, B., et al.: The Drift Table: Designing for Ludic Engagement. In: CHI 2004, April 24-29. ACM 1-58113-703-6/04/0004, Vienna (2004)

9. Nintendo, Nintendo Wii, http://www.nintendo.co.uk/Wii/Wii-94559.html

10. Sony, PlayStationMove, Motion Controller, http://uk.playstation.com/psmove/

11. Microsoft, Kinect for Xbox, http: / /www . xbox.com/en-GB/Kinect

12. Broeren, J., Jalminger, J., Johansson, L.-Å., Parmerud, A., Pareto, L., Rydmark, M.: Information and communication technology - a person-centered approach to stroke care. In: Proc. 9th Intl Conf. Disability, Virtual Reality \& Associated Technologies, Laval, France (2012)

13. Chang, Y.-J., Chen, S.-F., Huang, J.-D.: A Kinect-based system for physical rehabilitation: a pilot study for young adults with motor disabilities. J. Research in Developmental Disabilities 32(6), 2566-2570 (2011)

14. Lange, B., Chang, C.Y., Suma, E., Newman, B., Rizzo, A.S., Bolas, M.: Development and evaluation of low cost game-based balance rehabilitation tool using the Microsoft Kinect sensor. In: Proc. IEEE Conf. Eng. Med. Biol. Soc., pp. 2011:1831-2011:1834 (2011)

15. Shires, L., Battersby, S., Lewis, J., Brown, D., Sherkat, N., Standen, P.: Enhancing the tracking capabilities of the Microsoft Kinect for Stroke Rehabilitation. In: IEEE 2nd International Serious Games and Applications for Health (SeGAH), Portugal, pp. 1-8 (May 2013) 
16. Deterding, S., Dixon, D., Khaled, R., Nacke, L.: From Game Design Elements to gamefulness: Defining "Gamification”. In: MindTrex 2011, Tampere, Finland (September 28030, 2011)

17. Microsoft, Kinect Effect - Rehabilitating with Kinect I Royal Berkshire Hospital, England, XboxViewTV, http: / /www . youtube. com/watch?v=5sv3nKPeM9g

18. Simmons, S., McCrindle, R., Sperrin, M., Smith, A.: Prescription Software for Recovery and Rehabilitation using Microsoft Kinect. In: Proc. 7th International Conference on Pervasive Computing Technologies for Healthcare (Pervasive Health), Venice, pp. 323-326 (May 2013) 